Even more striking is the description of a mimosa which

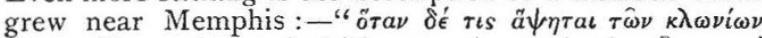

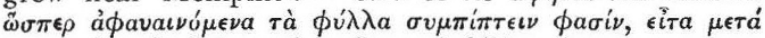

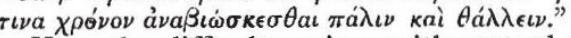

Here the difficulty arises with regard to the species which is denoted, but special investigation by Dr. Schweinfurth elicited the information that in the vicinity there grows Mimosa asperata, a plant the sensitivity of which is almost unknown to botanists. Another graphic description is that of the banyan, $\sigma \nu \kappa \hat{\eta}$ 'I $\Sigma \delta \kappa \hat{\eta}$, with the allusion to the roots developed from the branches, which are roots because they are lighter in colour and leafless. But the book contains many similar points of interest, and Dr. Bretzl has furnished abundant proof of the accuracy of perception and faculty of discernment possessed by some of the ancient Greeks.

The sources of information are to be traced to the memoirs of certain of Alexander's retinue. These manuscripts, which were deposited in Babylon, have unfortunately been lost; but they were apparently available to Theophrastus, who has worked up the material with truly remarkable intuition. Between the writings of Theophrastus and those of other authors, notably Pliny, Dr. Bretzl draws a sharp line of distinction, the distinction, in fact, between the original thinker and the annotator.

Practical Plane and Solid Geometry for Elementary Students. By Joseph Harrison. Pp. xiii +250. (London: Macmillan and Co., Ltd., I903.) Price 2s. $6 d$.

This little book will be found very useful for the teaching of the fundamental principles of geometry to young students. The most important properties of triangles and other plane figures are illustrated by means of accurate drawing and numerical calculation, and thus appeal more readily to the understanding and memory than if the beginner were made acquainted with them by means of the severe and tedious logic of Euclid. The great advantage of such a book as this is that it prepares the mind of the beginner for methods of accurate logical demonstration at a later stage in his studies. The very large number of numerical exercises requiring calculation and the use of instruments should suffice to give the student a very firm knowledge of all the important part of elementary geometry; and for this reason the book can be confidently recommended to teachers.

The first ten chapters are of this useful kind; then follow some chapters on the nature of vectors and their addition, including some properties of uniplanar forces acting on a particle the necessity for which may, perhaps, be doubted. In these chapters we meet with a little careless writing which, doubtless, will be corrected in the next edition. Thus, the first sentence (or what should be a sentence) on p. II 8 reminds us of Mr. Skae's item in "The Jumping Frog ": a verb is missing and no assertion is made. The use of the expression " in tandem, or follow-myleader" to indicate cyclic order in the sides of a triangle is of doubtful propriety; but such trifles constitute, of course, no serious objection.

The notation $l_{\theta}$ for the magnitude and direction of a vector (p. 130) is distinctly useful in the composition of vectors. Chapter xiii., on concurrent forces, will, of course, be omitted by the beginner whose aim is to acquire only a knowledge of the elements of geometry; and it scarcely belongs to the subject.

The remaining five chapters deal with geometrical drawing in three dimensions, and they constitute a very good introduction to the subject, the figures being very numerous, and accompanied by a large number of numerical examples.

No. $176 \mathrm{I}$, voL. 68$]$
Die Aluminium-Industrie. By Dr. F. Wintelen. Pp. xi+ 108. (Braunschweig: Friedrich Vieweg und Sohn, I9o3.) Price 6 marks.

THIs very interesting monograph upon the aluminium industry commences with a short historical introduction, in which we learn that Davy, so far back as 1808 , after he had discovered sodium and potassium, endeavoured to prepare aluminium by electrolysing alumina. In this he was not successful, and it fell to the lot of Wöhler in 1827 first to prepare the metal by purely chemical methods. Bunsen, however, was able in 1854 to obtain it by electrolysing its chloride. In a table on p. 5 the variation in the price of the metal is traced since 1854 , when it was merely a chemical curiosity. Its value in that year was $120 l$. per kilo, and even in 1889 it cost 2 l.; but with the improvements of the electrical methods, the price rapidly dropped, until in Igor it ranged from $2 s$. to 2s. $6 d$. per kilo. Following the historical portion of the work, a very full account of the physical and chemical properties of the metal is given. It is not until we reach p. 22 that the present methods of obtaining the metal are gone into, but here the thoroughness of the treatment leaves nothing to be desired. In the first place a careful account of the preparation of the outgoing materials used in the manufacture is given. This part of the work is of very considerable value. Everyone is aware that bauxite and cryolite are the substances used for preparing aluminium, and those who have studied the subject know that these substances cannot, as a rule, be employed without being first purified. In this book the methods of purification are described in detail, and methods of analysis are also set forth. Page 54 is headed "carbon electrodes"; these are employed both for the anode and kathode, in consequence of impurities introduced into the bath when other electrodes are used. The author gives details of the manufacture of these carbon electrodes-ten pages are devoted to this. Some useful diagrams illustrating the way in which the electrodes become corroded during the electrolysis are also given.

The last few pages of the monograph are devoted to the "working up of the metal"; one of the most interesting points being the method for welding the metal which is employed by Heräus, of Hanau. It consists in heating aluminium sheets with a hydrogen flame to a temperature of about $400^{\circ}$. The edges are then pressed together, and after being worked for some time with the hammer, they weld together in such a manner that tubes made in this way can hardly be distinguished from seamless ones.

This monograph is one of the most interesting and useful which we have had the pleasure of reading for a long time. The facts are well arranged, and although there are 108 pages devoted to the single subject of the aluminium industry, we do not consider that the work suffers from prolixity; we wish this could be said of many German monographs which have lately been published.

F. M. P.

\section{Die Konstitution des Kamphers und seiner wichtigsten}

Derivate. By Ossian Aschan. Pp. xi+117. (Braunschweig: Friedrich Vieweg und Sohn, rgo3.) Price 3.50 marks.

THE chemistry of camphor and its derivatives has occupied the attention of chemists for many years, and has now become so specialised that it is almost impossible for the ordinary chemist to keep up with the immense amount of research published in the journals devoted to chemistry. The monograph by Prof. Aschan is accordingly very welcome, and will be found useful not only by the non-camphor chemist, but also 